\title{
Crystallographic and Spectral Equivalence of Boron-Carbide Polymorphs
}

\author{
Cody Kunka, Amnaya Awasthi, Ghatu Subhash* \\ University of Florida, Gainesville, FL 32611 USA, *subhash@ufl.edu
}

\begin{abstract}
The extraordinary properties of boron carbide originate from the collection of polymorphs that comprise fabricated samples. However, traditional nomenclature that differentiates and describes these crystal structures often restricts the ability to fully model variations in material properties and performance. To highlight and transcend these limitations, this study introduces a modified nomenclature and defines new groups of equivalent polymorphs based on quantum-mechanical simulations. Trends in lattice parameters, total energy, and Raman spectra are explained in terms of the unique covalent-bonding environments of boron-carbide polymorphs.
\end{abstract}

Keywords. Boron Carbide, Raman Spectra, Crystallography, Equivalence, Density Functional Theory

For boron carbide, polymorphism affords large variability in both carbon percentage (from 8.0 to 20 at $\%$ ) and atomic arrangement to strongly influence physical, mechanical, and electronic properties [1-11]. Further, closeness of formation energies of these crystal structures induces many polymorphs to form in the same fabricated sample [10].

Unfortunately, limitations in experimental techniques and naming conventions obscure the identity and distribution of these crystal structures. Hence, the current investigation introduces a highly specific nomenclature and models polymorphs through quantum-mechanical simulations.

The simplest unit cell for each boron-carbide polymorph comprises a three-atom chain and a twelve-atom icosahedron [see Fig. 1(a)]. Within each icosahedron, six polar $(p)$ atoms connect multiple icosahedra directly, and six equatorial (e) atoms connect multiple icosahedra through the chains [see Figs. 1(b) and 1(c)]. Traditionally [1], researchers differentiate boron-carbide crystal structures with the following notation: $\left(\mathrm{B}_{\mathrm{m}} \mathrm{C}_{\mathrm{nfo}}\right)$ ijk where $\left(\mathrm{B}_{\mathrm{m}} \mathrm{C}_{\mathrm{nfo}}\right)$ represents the icosahedron (sites 1 -- 12), and ijk represents the chain (sites i, j, and k) [see Fig. 1(a)]. Subscripts "m" and " $n$ " indicate the number of boron atoms and carbon atoms, respectively, in the icosahedron. If $n=0, C_{n f o}$ is excluded. Subscript " $\mathrm{f}$ " marks the family of the icosahedral carbons as either polar $(p)$ or equatorial $(e)$. If $\mathrm{n}=2$ and $\mathrm{f}=p$, "o" is often included to describe relative orientation of polar carbons [1]. The simplest example of this traditional nomenclature is $\left(\mathrm{B}_{12}\right) \mathrm{CCC}$, which has a CCC chain and a twelve-boron icosahedron. Here, the six equatorial boron atoms represent one $6 h$ Wyckoff site of space group $166(R \overline{3} \mathrm{~m})$, and the six polar boron atoms represent another [12,13]. Thus, there is no 
need to differentiate within polar sites or within equatorial sites for $\left(\mathrm{B}_{12}\right) \mathrm{CCC}$. However, an icosahedron with a carbon atom [i.e., $\left(\mathrm{B}_{11} \mathrm{C}_{1 \mathrm{f}}\right) \mathrm{ijk}$ ] no longer exhibits the aforementioned symmetry. Depending on chain composition, location of this icosahedral carbon may need to be explicitly specified in the nomenclature to differentiate unique crystal structures. Adding a second icosahedral carbon atom poses similar issues. Consider $\left(\mathrm{B}_{10} \mathrm{C}_{2 \mathrm{p} \text {,antipodal }}\right) \mathrm{BCB}$, which has one carbon atom in polar site 1,2, or 3 and another carbon atom in the polar site 4, 5, or 6 . Because the distance between pairs of antipodal atoms varies, additional specificity in the nomenclature is required to describe variability in properties.

Comprehensive understanding of material performance and full tailoring of properties mandate differentiation of all unique polymorphs. Unfortunately, the commonly adopted nomenclature does not always differentiate polymorphs with the same stoichiometry. Further, while fabricated boron carbide may exhibit $R \overline{3} m$ symmetry on average, constituent polymorphs may not. For these reasons, this manuscript modifies nomenclature from $\left(\mathrm{B}_{\mathrm{m}} \mathrm{C}_{\mathrm{nfo}}\right)$ ijk to $\left(\mathrm{B}_{\mathrm{m}} \mathrm{C}_{\mathrm{fz}}\right) \mathrm{ijk}$. Here, " $\mathrm{z}$ " specifies location(s) of icosahedral carbon atom(s) in the unit cell according to Fig. 1(a). Reduced coordinates may slightly change with position and/or number of carbon atoms. The " $\mathrm{C}_{\mathrm{fz}}$ " is excluded if no icosahedral carbons are present [e.g., $\left(\mathrm{B}_{12}\right) \mathrm{CCC}$, and site numbers are separated by commas if multiple icosahedral carbons are present [e.g., $\left(\mathrm{B}_{10} \mathrm{C}_{\mathrm{p} 2,5}\right) \mathrm{BCB}$. Subscript "f" is retained to avoid confusing " $\mathrm{z}$ " (site number) for " $\mathrm{n}$ " (number of atoms). Unlike traditional nomenclature, this modified scheme can model differences due to icosahedral carbon position within polar sites and within equatorial sites [e.g., $\left(\mathrm{B}_{11} \mathrm{C}_{\mathrm{p} 1}\right) \mathrm{BCC}$ vs. $\left(\mathrm{B}_{11} \mathrm{C}_{\mathrm{p} 4}\right) \mathrm{BCC}$ ] and can also differentiate a chain from its mirror [e.g., $\left(\mathrm{B}_{11} \mathrm{C}_{\mathrm{p} 1}\right) \mathrm{BCC}$ vs. $\left(\mathrm{B}_{11} \mathrm{C}_{\mathrm{p} 1}\right) \mathrm{CCB}$. Hence, this new nomenclature lays a more robust foundation for understanding polymorphism and the corresponding variation in properties.

The current study employs ABINIT [14], a popular atomistic solver for density functional theory (DFT), to model all fifty-two variations of boron carbide commonly proposed in both experimental and theoretical investigations on boron carbide [1,8,10,15-17] except those with chain vacancies [18-21] or without Raman activity [11,22]. Single unit cells [Fig. 1(a)] with periodic-boundary conditions, a Monkhorst $k$-point mesh, and norm-conserving pseudopotentials with the Troullier-Martins scheme and Pulay mixing are utilized. The exchange-correlation functional is evaluated by both the Teter-Pade local-density approximation (LDA) [23] and the Perdew-Burke-Ernzerhof generalized-gradient approximation (GGA) [24]. From this energy-minimization process (accurate up to $10^{-14} \mathrm{eV}$ ), lattice parameters, mass densities, and relative energies per atom are calculated (see Table I). Structures with the same properties are considered an "equivalentlattice group," assigned a Roman numeral, and ordered by energy relative that of group i. All calculated lattice parameters 
and energies from the seven $\mathrm{B}_{4} \mathrm{C}$ polymorphs modeled in a previous DFT study [9] have been replicated within $0.3 \%$ error in this investigation.

Two competing effects seem to drive the ordering of the polymorphs by energy in Table I. First, small distances between carbon atoms generally were found to correlate with large energies. Carbon has higher electronegativity than boron, so spatially concentrating carbon atoms concentrates charge and raises potential energy. Hence, groups i and ii (CBC chain) have lower energy than groups iii - vii (CCC, BCC, or CCB chain). Groups i (0 meV) and iii (166 meV) have lower energy than groups ii $(36 \mathrm{meV})$ and iv $(174 \mathrm{meV})$, respectively, because the icosahedral carbon of the former two groups are not directly bound to chain carbons as in the latter two groups. Likewise, group viii (304 meV) has lower energy than group $\mathrm{x}(346 \mathrm{meV})$ because the icosahedral carbons are bonded to icosahedral borons in the former but to icosahedral carbons in the later [see Fig. 1(b)]. Second, the number of icosahedral carbon atoms was found to correlate with energy. That $\left(\mathrm{B}_{12}\right)$ is the lowest-energy icosahedron is consistent with the fact that boron icosahedra can form without chains $[25,26]$. Energy increases from group iii (zero icosahedral carbons) to groups iv - vii (one icosahedral carbon) to groups viii - $\mathrm{x}$ (two icosahedral carbons). Polymorphs with a CBC chain and a single icosahedral carbon atom (groups $\mathrm{i}$ and ii) have lower energy than $\left(\mathrm{B}_{12}\right) \mathrm{CCC}$ (group iii) simply as a result of the competition of these two effects. In group iii, the rise in energy due to direct bonding of the carbons in the CCC chains supersedes the low energy of the $\left(\mathrm{B}_{12}\right)$ icosahedron. In groups i and ii, however, the chain carbons are favorably separated by a boron atom.

After fully relaxing lattice parameters and atomic positions, density functional perturbation theory (DFPT) in ABINIT [27] is employed to calculate natural frequencies, phonon eigenvectors, and dielectric tensors. Intensities of the Raman-active natural frequencies can then be computed from a well-established post-processing method [28]. Because this manuscript seeks to establish the equivalencies of polymorphs, Raman-peak widths, which result from anharmonic effects [29], are beyond the scope of this study and are therefore not included. With a temperature of $300 \mathrm{~K}$ and an excitation wavelength of $532 \mathrm{~nm}$, this analysis provides the first high-fidelity simulations for numerous boron-carbide polymorphs. Each row of Table II lists the top-five Raman peaks (natural frequency; normalized intensity) for polymorphs that exhibit identical Raman spectra. Most importantly, these "equivalent-Raman groups" are found identical to the equivalent-lattice groups and are therefore labeled in the same fashion as in Table I. Agreement in these equivalencies is due to the fact that both energy and Raman activity originate from local bonding environment (see previous rationalization of energy trends and [30]). Also, this agreement suggests that these equivalent groups may share other properties as well. 
Because LDA and GGA produced similar values and identical trends (see Tables I and II), Fig. 2 plots representative LDA Raman spectra to illustrate salient points. Figure 2(a) compares the simulated Raman peaks from group i with an experimental spectrum from hot-pressed boron carbide. For the first time, there is strong agreement of most peak locations and intensities between simulated and experimental spectra. This agreement suggests that group-i polymorphs represent the majority of fabricated boron carbide. This finding is consistent with many prior studies $[8,10,15,16]$ but offers new information on the vibrational properties of this popular crystal structure. Hot-pressed boron carbide contains several components (e.g., free carbon, boron clusters, and boron-carbide polymorphs of possibly different stoichiometries), so peaks not represented by the group-i spectrum are likely due to other species. For example, the relatively large experimental peaks around $980 \mathrm{~cm}^{-1}$ may be due to group viii or group x [see Table II and Fig. 2(b)]. This finding is consistent with prior literature $[8,16]$ that implicated the $\left(\mathrm{B}_{10} \mathrm{C}_{2 \mathrm{p}, \text { antipodal }}\right) \mathrm{BCB}$ traditional family as the largest minority constituent. Figures 2(b) and 2(c) show variability in Raman spectrum for polymorphs in the same traditional families. The former highlights the need to differentiate antipodal configurations, and the latter highlights the need to differentiate chains from their mirrors (i.e., ijk vs. kji). These shortcomings of the traditional nomenclature have been resolved by the new nomenclature presented in this investigation.

By modeling all members of traditional families, this investigation offers the first comprehensive description of the $\mathrm{B}_{4} \mathrm{C}$ crystal structures and their properties. For example, Goddard [31-33] modeled amorphization of boron carbide based on slip systems, energies, and twins of a single polymorph from the $\left(B_{11} C_{p}\right) C B C$ traditional family. The current investigation found all six polymorphs in this traditional family identical (see group i in Tables I and II), so the assumption of those works is valid. On the other hand, Fanchini [10] and Aydin [9] modeled one polymorph from each traditional family with a BCC chain to help explain the kinetics of amorphization and the variability in hardness, respectively. The current investigation has shown that these traditional families exhibit significant variation in energy and Raman spectrum (see groups iv - vii in Tables I and II), so the results of those studies may be incomplete. Understanding amorphization is paramount for improving the performance of boron carbide [34-40], so the current results may prove especially useful.

By introducing a new nomenclature and by simulating lattice parameters, energy, and Raman spectra, this manuscript has improved the understanding of boron-carbide crystallography. Most importantly, these simulations have enabled novel classifications of polymorphs with differences in occupancy in the polar/equatorial sites or with differences 
in chain orientation. These distinctions were not previously possible due to limitations in the old nomenclature and serve

to highlight the importance of understanding polymorphism-induced variability in local bonding environment.

Comprehensive understanding of the structure, properties, and promise of this advanced ceramic mandates these

improvements.

\section{CAPTIONS}

FIG 1. (a) Simplest boron-carbide unit cell color-coded by site family: polar (red, sites 1 -- 6), equatorial (blue, sites 7 -- 12), and chain (black, sites $i, j$, and $k$ ). (b) Connectivity of chain atoms. (c) Nearest neighbors of icosahedral atoms.

TABLE I. Lattice parameters, mass densities, and relative energies per atom of B4C (20 at\%) polymorphs for the LDA (GGA). Structures with the same energy and combination of lattice parameters are listed on the same row and assigned a Roman numeral.

TABLE II. Natural frequencies $(\omega)$ and normalized intensities (I) for the dominant Raman peaks of each equivalent group of $B_{4} C(20$ at\% C) polymorphs for the $L D A(G G A)$.

FIG. 2. (a) Strong agreement of Raman spectra from group-i simulations (LDA) and experimentally (EXP) hot-pressed boron carbide. $(b, c)$ Variation within $\left(B_{10} C_{2 p, \text { antipodal }}\right) B C B,\left(B_{11} C_{e}\right) B C C$ and $\left(B_{11} C_{e}\right) C C B$ and traditional families.

\section{ACKNOWLEDGEMENTS}

This material is based upon work supported by the National Science Foundation Graduate Research Fellowship under Grant No. DGE-1315138 and by the Army Research Office under Grant No. ARO-W911NF-14-1-0230. This work used the Extreme Science and Engineering Discovery Environment (XSEDE), which is supported by National Science Foundation grant number ACI-1053575. Startup resources were used under TG-MSS15006. The present results have been obtained through the use of the ABINIT code, a common project of the Université Catholique de Louvain, Corning Incorporated, the Université de Liège, the Commissariat à l'Energie Atomique, Mitsubishi Chemical Corp., the Ecole Polytechnique Palaiseau, and other contributors (http://www.abinit.org). The authors also thank Dr. Aleksandr Chernatynskiy for his assistance in DFT modeling.

\section{REFERENCES}

[1] V. Domnich, S. Reynaud, R. Haber, M. Chhowalla, J. Am. Ceram. Soc. 94 (2011) 3605. doi:10.1111/j.1551-2916.2011.04865.x.

[2] F. Thevenot, J. Eur. Ceram. Soc. 6 (1990) 205. doi:10.1016/0955-2219(90)90048-K.

[3] S. Konovalikhin, V. Ponomarev, J. Phys. Chem. 84 (2010) 1445. doi:10.1134/S0036024410080297.

[4] D. Taylor, J. McCauley, T. Wright, J. Phys. Condens. Matter. 24 (2012). doi:10.1088/0953-8984/24/50/505402.

[5] G. Kwei, B. Morosin, J. Phys. Chem. 100 (1996) 8031. doi:10.1021/jp953235j.

[6] R. McCuiston, J. Lasalvia, J. McCauley, W. Mayo, Adv. Cer. Armor IV. 29 (2009) 153.

[7] D. Wang, Q. Yan, B. Wang, Y. Wang, J. Yang, G. Yang, J. Chem. Phys. 140 (2014). doi:10.1063/1.4882071.

[8] N. Vast, J. Sjakste, E. Betranhandy, Journal of Physics: Conference Series. 176 (2009) 012002 (18 pp.). doi:10.1088/17426596/176/1/012002.

[9] S. Aydin, M. Simsek, Phys. Status Solidi B. 246 (2009) 62. doi:10.1002/pssb.200844328.

[10] G. Fanchini, J. McCauley, M. Chhowalla, Phys. Rev. Lett. 97 (2006). doi:10.1103/PhysRevLett.97.035502.

[11] S. Mondal, E. Bykova, S. Dey, S.I. Ali, N. Dubrovinskaia, L. Dubrovinsky, G. Parakhonskiy, S. van Smaalen, Scientific Reports. $6(2016) 19330$.

[12] M. Aroyo, J. Perez-Mato, C. Capillas, E. Kroumova, S. Ivantchev, G. Madariaga, A. Kirov, H. Wondratschek, Zeitschrift für Kristallographie, Z. Krist. 221, 1 (2006) 15-27.

[13] O. Sologub, Y. Michiue, T. Mori, Acta Crystallographica, Section E (Structure Reports Online). E68 (2012) 67. doi:10.1107/S1600536812033132.

[14] X. Gonze, B. Amadon, P.-. Anglade, J.-. Beuken, F. Bottin, P. Boulanger, F. Bruneval, D. Caliste, R. Caracas, M. Cote, T. Deutsch, L. Genovese, P. Ghosez, M. Giantomassi, S. Goedecker, D.R. Hamann, P. Hermet, F. Jollet, G. Jomard, S. Leroux, M. Mancini, S. Mazevet, M.J.T. Oliveira, G. Onida, Y. Pouillon, T. Rangel, G.-. Rignanese, D. Sangalli, R. Shaltaf, M. Torrent, M.J. Verstraete, G. Zerah, J.W. Zwanziger, Comput. Phys. Commun. 180 (2009) 2582. doi:10.1016/j.cpc.2009.07.007. 
[15] R. Lazzari, N. Vast, J. Besson, S. Baroni, A. Dal Corso, Phys. Rev. Lett. 83 (1999) 3230. doi:10.1103/PhysRevLett.83.3230.

[16] F. Mauri, N. Vast, C. Pickard, Phys. Rev. Lett. 87 (2001) 085506. doi:10.1103/PhysRevLett.87.085006.

[17] H. Werheit, J. Phys. Condens. Matter. 19 (2007) 14 pp. doi:10.1088/0953-8984/19/18/186207.

[18] E. Betranhandy, N. Vast, J. Sjakste, Solid State Sciences. 14 (2012) 1683. doi:10.1016/j.solidstatesciences.2012.07.002.

[19] J. Saal, S. Shang, L. Zi-Kui, Appl. Phys. Lett. 91 (2007) 231915. doi:10.1063/1.2818661.

[20] R. Raucoules, N. Vast, E. Betranhandy, J. Sjakste, Physical Review B (Condensed Matter and Materials Physics). 84 (2011) 014112 (5 pp.). doi:10.1103/PhysRevB.84.014112.

[21] A. Jay, N. Vast, J. Sjakste, O.H. Duparc, Appl. Phys. Lett. 105 (2014). doi:10.1063/1.4890841.

[22] K. Shirai, K. Sakuma, N. Uemura, Phys. Rev. B. 90 (2014) 064109 (10 pp.). doi:10.1103/PhysRevB.90.064109.

[23] S. Goedecker, M. Teter, J. Hutter, Phys. Rev. B. 54 (1996) 1703.

[24] J.P. Perdew, K. Burke, M. Ernzerhof, Phys. Rev. Lett. 77 (1996) 3865.

[25] X. Yan, W. Li, T. Goto, M. Chen, Appl. Phys. Lett. 88 (2006). doi:10.1063/1.2189826.

[26] D. Ghosh, G. Subhash, H.L. Chee, K.Y. Yoke, Appl. Phys. Lett. 91 (2007) 061910. doi:10.1063/1.2768316.

[27] X. Gonze, M. Verstraete, C. Audouze, M. Torrent, F. Jollet, AIP Conference Proceedings. 1504 (2012) 944. doi:10.1063/1.4771852.

[28] R. Caracas, E. Bobocioiu, Am. Mineral. 96 (2011) 437-443.

[29] R. Caracas, E.J. Banigan, Phys. Earth Planet. Inter. 174 (2009) 113. doi:10.1016/j.pepi.2009.01.001.

[30] K. Shirai, S. Emura, Journal of Physics: Condensed Matter. 8 (1996) 10919. doi:10.1088/0953-8984/8/50/018.

[31] Q. An, W. Goddard, T. Cheng, Phys. Rev. Lett. 113 (2014). doi:10.1103/PhysRevLett.113.095501.

[32] Q. An, W. Goddard, Phys. Rev. Lett. 115 (2015) 105501 (5 pp.). doi:10.1103/PhysRevLett.115.105501.

[33] K. Xie, Q. An, M. Toksoy, J. McCauley, R. Haber, W. Goddard, K. Hemker, Phys. Rev. Lett. 115 (2015) 175501 (5 pp.). doi:10.1103/PhysRevLett.115.175501.

[34] M. Chen, J. McCauley, K. Hemker, Science. 299 (2003) 1563. doi:10.1126/science.1080819.

[35] X. Yan, Z. Tang, L. Zhang, J. Guo, C. Jin, Y. Zhang, T. Goto, J. McCauley, M. Chen, Phys. Rev. Lett. 102 (2009). doi:10.1103/PhysRevLett.102.075505.

[36] K. Reddy, A. Hirata, P. Liu, T. Fujita, T. Goto, M. Chen, Scr. Mater. 76 (2014) 9. doi:10.1016/j.scriptamat.2013.12.001.

[37] K. Reddy, P. Liu, A. Hirata, T. Fujita, M. Chen, Nature. 4 (2013) 2483 (5 pp.). doi:10.1038/ncomms3483.

[38] S. Aryal, P. Rulis, W.Y. Ching, Physical Review B (Condensed Matter and Materials Physics). 84 (2011) 184112 (12 pp.). doi:10.1103/PhysRevB.84.184112.

[39] G. Subhash, D. Ghosh, J. Blaber, J.Q. Zheng, V. Halls, K. Masters, Acta Mater. $61 \quad(2013) 3888$. doi:10.1016/j.actamat.2013.03.028.

[40] D. Ghosh, G. Subhash, J.Q. Zheng, V. Halls, J. Appl. Phys. 111 (2012) 063523 (9 pp.). doi:10.1063/1.3696971. 


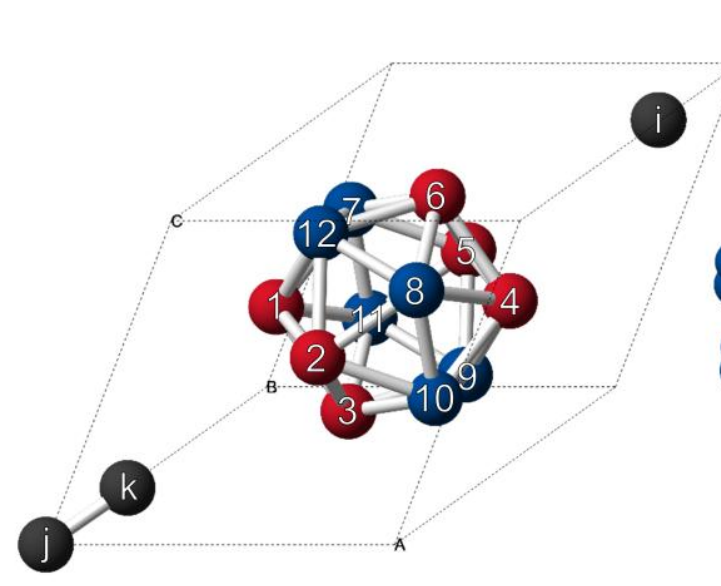

(a)

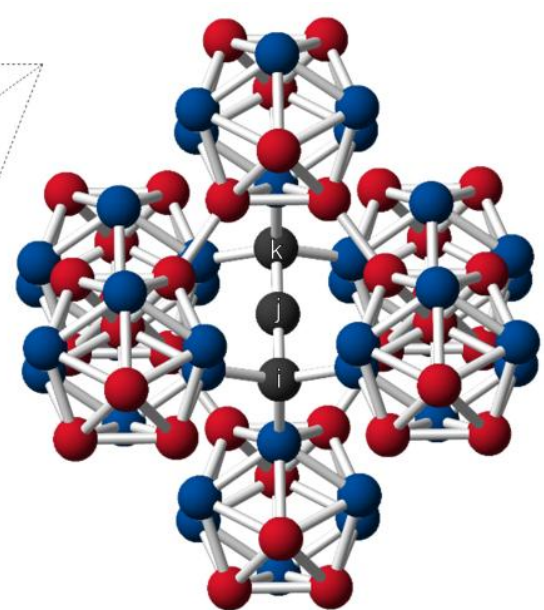

(b)

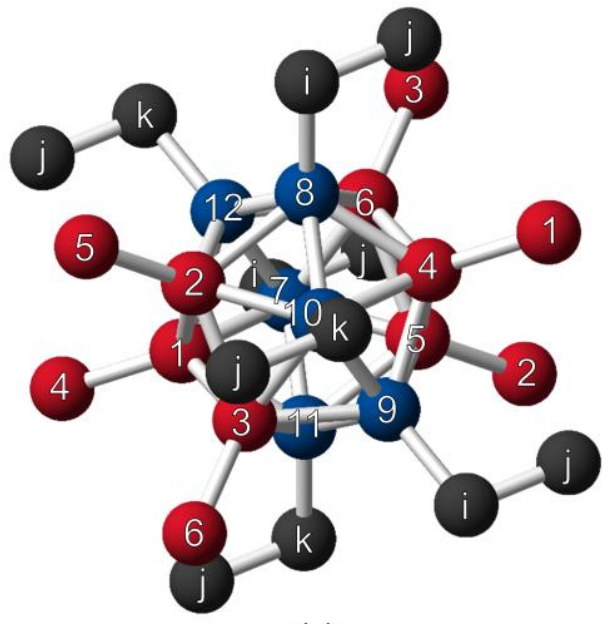

(c)

Figure 1 
Equivalence of $\mathrm{B}_{4} \mathrm{C}$ Polymorphs

Kunka, et al. 8
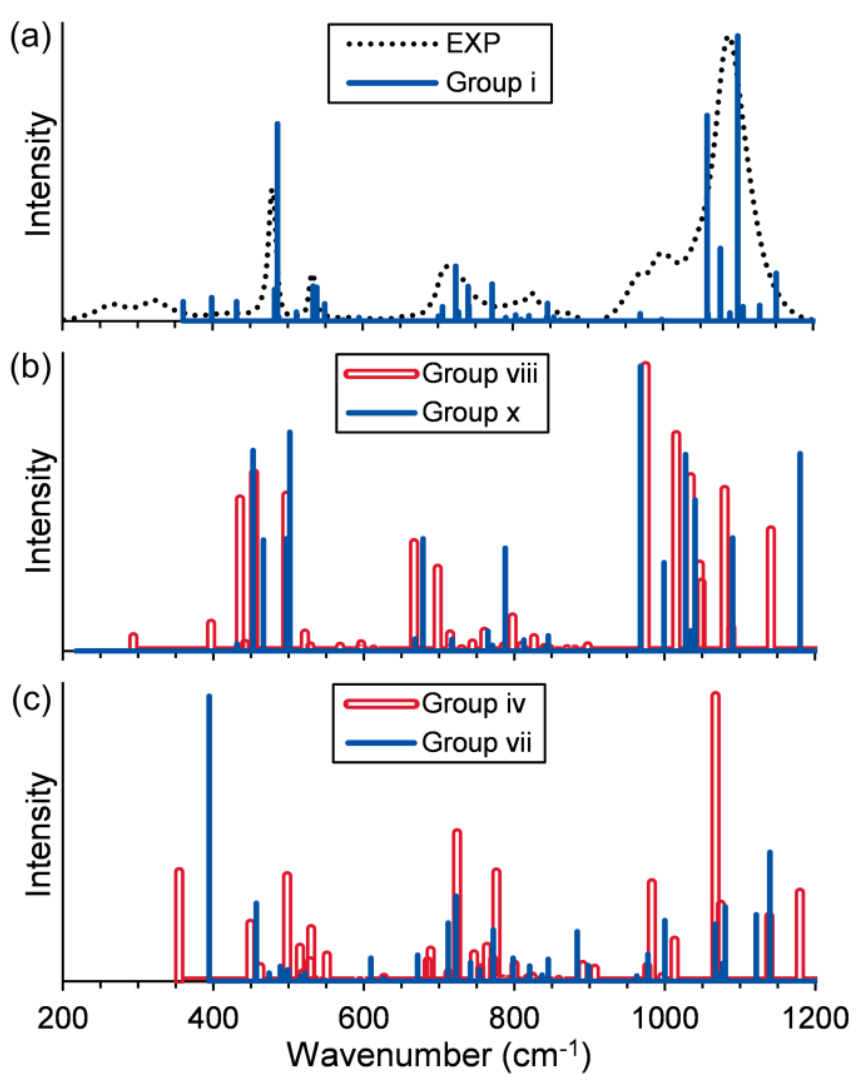

Figure 2 
TABLE I. Lattice parameters, mass densities, and relative energies per atom of B4C (20 at\%) polymorphs for the LDA (GGA). Structures with the same energy and combination of lattice parameters are listed on the same row and assigned a Roman numeral.

\begin{tabular}{|c|c|c|c|c|}
\hline $\begin{array}{c}\text { Group } \\
-- \\
\end{array}$ & $\begin{array}{l}\text { Equivalent B4C Polymorph(s) } \\
\text { zijk of }\left(\mathrm{B}_{\mathrm{m}} \mathrm{C}_{\mathrm{fz}}\right) \text { ijk }\end{array}$ & $\begin{array}{c}\text { Permutation of Lattice Parameters } \\
\AA\end{array}$ & $\begin{array}{l}\text { Mas Density } \\
\mathrm{g} / \mathrm{cm}^{3}\end{array}$ & $\begin{array}{c}\text { Rel. Energy } \\
\mathrm{meV}\end{array}$ \\
\hline $\mathrm{i}$ & $1 \mathrm{CBC}|2 \mathrm{CBC}| 3 \mathrm{CBC}|4 \mathrm{CBC}| 5 \mathrm{CBC} \mid 6 \mathrm{CBC}$ & $\{5.00,5.16,5.16\}(\{5.04,5.19,5.19\})$ & $2.60(2.56)$ & $0(0)$ \\
\hline ii & $7 \mathrm{CBC}|8 \mathrm{CBC}| 9 \mathrm{CBC}|10 \mathrm{CBC}| 11 \mathrm{CBC} \mid 12 \mathrm{CBC}$ & $\{5.11,5.15,5.15\}(\{5.15,5.18,5.18\})$ & $2.59(2.55)$ & $36(36)$ \\
\hline iii & $\mathrm{CCC}$ & $\{5.14,5.14,5.14\}(\{5.17,5.17,5.17\})$ & $2.55(2.50)$ & $77(71)$ \\
\hline iv & $7 \mathrm{BCC}|10 \mathrm{CCB}| 8 \mathrm{BCC}|11 \mathrm{CCB}| 9 \mathrm{BCC} \mid 12 \mathrm{CCB}$ & $\{5.05,5.16,5.16\}(\{5.10,5.19,5.19\})$ & $2.55(2.50)$ & $166(161)$ \\
\hline $\mathrm{v}$ & $4 \mathrm{BCC}|1 \mathrm{CCB}| 5 \mathrm{BCC}|2 \mathrm{CCB}| 6 \mathrm{BCC} \mid 3 \mathrm{CCB}$ & $\{4.98,5.17,5.17\}(\{5.02,5.20,5.20\})$ & $2.54(2.50)$ & $169(164)$ \\
\hline vi & $1 \mathrm{BCC}|4 \mathrm{CCB}| 2 \mathrm{BCC}|5 \mathrm{CCB}| 3 \mathrm{BCC} \mid 6 \mathrm{CCB}$ & $\{4.96,5.17,5.17\}(\{5.00,5.20,5.20\})$ & $2.55(2.50)$ & $174(169)$ \\
\hline vii & $10 \mathrm{BCC}|7 \mathrm{CCB}| 11 \mathrm{BCC}|8 \mathrm{CCB}| 12 \mathrm{BCC} \mid 9 \mathrm{CCB}$ & $\{5.10,5.16,5.16\}(\{5.13,5.19,5.19\})$ & $2.53(2.49)$ & 225 (219) \\
\hline viii & $3,5 \mathrm{BCB}|2,6 \mathrm{BCB}| 3,4 \mathrm{BCB}|1,6 \mathrm{BCB}| 1,5 \mathrm{BCB} \mid 2,4 \mathrm{BCB}$ & $\{5.01,5.01,5.21\}(\{5.05,5.05,5.24\})$ & $2.54(2.59)$ & 304 (299) \\
\hline ix & $5,6 \mathrm{BCB}|2,3 \mathrm{BCB}| 4,6 \mathrm{BCB}|1,3 \mathrm{BCB}| 4,5 \mathrm{BCB} \mid 1,2 \mathrm{BCB}$ & $\{5.01,5.01,5.23\}(\{5.05,5.05,5.25\})$ & $2.52(2.47)$ & $328(322)$ \\
\hline $\mathrm{x}$ & $1,4 \mathrm{BCB}|2,5 \mathrm{BCB}| 3,6 \mathrm{BCB}$ & $\{4.81,5.22,5.22\}(\{4.85,5.25,5.25\})$ & $2.55(2.50)$ & $346(342)$ \\
\hline
\end{tabular}

TABLE II. Natural frequencies $(\omega)$ and normalized intensities (I) for the dominant Raman peaks of each equivalent group of $B_{4} C$ (20 at\% C) polymorphs for the $L D A(G G A)$.

\begin{tabular}{cccccc} 
Group & Raman Peak \#1 & Raman Peak \#2 & Raman Peak \#3 & Raman Peak \#4 \\
-- & $\omega$ in $\mathrm{cm}^{-1} ; \mathrm{I}$ & $\omega$ in $\mathrm{cm}^{-1} ; \mathrm{I}$ & $\omega$ in $\mathrm{cm}^{-1} ; \mathrm{I}$ & $\begin{array}{c}\text { Raman Peak \#5 } \\
\omega \mathrm{cm}^{-1} ; \mathrm{I}\end{array}$ \\
\hline \hline i & $1099 ; 1.00(1019 ; 1.00)$ & $1058 ; 0.72(1074 ; 0.97)$ & $486 ; 0.69(480 ; 0.85)$ & $1076 ; 0.25(1044 ; 0.26)$ & $724 ; 0.19(1116 ; 0.24)$ \\
ii & $1082 ; 1.00(1054 ; 1.00)$ & $1118 ; 0.60(1080 ; 0.58)$ & $512 ; 0.50(504 ; 0.54)$ & $747 ; 0.30(730 ; 0.34)$ & $703 ; 0.26(688 ; 0.29)$ \\
iii & $1160 ; 1.00(1154 ; 1.00)$ & $758 ; 0.87(743 ; 0.97)$ & $1098 ; 0.53(1059 ; 0.59)$ & $999 ; 0.37(968 ; 0.54)$ & $707 ; 0.08(683 ; 0.12)$ \\
iv & $1067 ; 1.00(1046 ; 1.00)$ & $724 ; 0.52(952 ; 0.61)$ & $355 ; 0.38(1153 ; 0.58)$ & $776 ; 0.38(754 ; 0.56)$ & $498 ; 0.37(1039 ; 0.55)$ \\
v & $1062 ; 1.00(1021 ; 1.00)$ & $1160 ; 1.00(1147 ; 0.73)$ & $377 ; 0.79(455 ; 0.60)$ & $460 ; 0.61(383 ; 0.55)$ & $1076 ; 0.42(1039 ; 0.34)$ \\
vi & $354 ; 1.00(1030 ; 1.00)$ & $1071 ; 0.89(369 ; 0.80)$ & $1685 ; 0.46(453 ; 0.47)$ & $460 ; 0.38(1144 ; 0.43)$ & $1164 ; 0.36(1673 ; 0.39)$ \\
vii & $394 ; 1.00(398 ; 1.00)$ & $1668 ; 0.58(1653 ; 0.66)$ & $1140 ; 0.45(737 ; 0.45)$ & $723 ; 0.30(1126 ; 0.41)$ & $457 ; 0.27(453 ; 0.36)$ \\
viii & $975 ; 1.00(978 ; 1.00)$ & $1015 ; 0.76(948 ; 0.94)$ & $454 ; 0.62(448 ; 0.65)$ & $1035 ; 0.61(431 ; 0.60)$ & $1080 ; 0.56(1045 ; 0.59)$ \\
ix & $264 ; 1.00(267 ; 1.00)$ & $409 ; 1.00(979 ; 0.54)$ & $348 ; 0.96(394 ; 0.53)$ & $1016 ; 0.91(345 ; 0.38)$ & $971 ; 0.75(944 ; 0.31)$ \\
x & $968 ; 1.00(993 ; 1.00)$ & $502 ; 0.77(942 ; 0.71)$ & $453 ; 0.70(446 ; 0.66)$ & $1028 ; 0.69(498 ; 0.62)$ & $1180 ; 0.69(1151 ; 0.53)$
\end{tabular}




\section{Graphical Abstract}
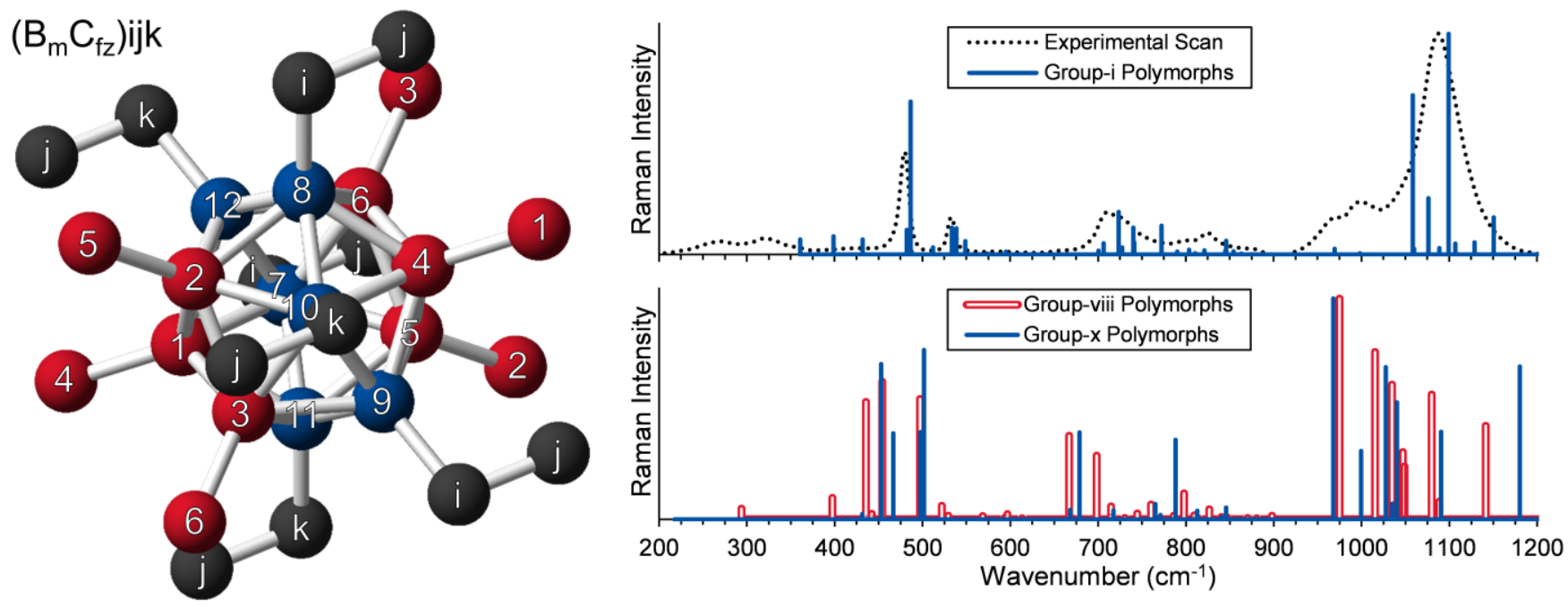